\title{
Superconducting Multiplexer Filter Bank for a Frequency-Selective Power Limiter
}

\author{
Eduard Rocas, Student Member, IEEE, Alberto Padilla, Jordi Mateu, Senior Member, IEEE, Nathan Orloff, \\ Juan M. O'Callaghan, Senior Member, IEEE, Carlos Collado, Senior Member, IEEE, and James C. Booth
}

\begin{abstract}
This work proposes a superconducting multiplexer filter bank configuration to be used as a frequency-selective power limiter. The proposed configuration limits narrowband high-power signals within a single frequency band without degrading the signal performance in the rest of the frequency bands. To accomplish this, we need to calculate the limiting power of superconducting filters implemented by means of half-wavelength transmission line resonators. The limiting power is obtained as a function of the resonator geometry, filter bandwidth, and filter order. Practical issues occurring in superconducting filters operating at high power, such as the reduction of the quality factors and de-tuning, are also analyzed and shown to not adversely affect the overall multiplexer power limiter performance.
\end{abstract}

Index Terms-Critical current, filter, filter bank, limiter, multiplexer.

\section{INTRODUCTION}

$\mathbf{O}$ $\mathrm{NE}$ of the fundamental properties of superconducting materials is their ability to switch from a high-loss normal state to a low-loss superconducting state, when cooled below the critical temperature $\mathrm{T}_{\mathrm{C}}$ [1]. But these materials, while in the superconducting state below $\mathrm{T}_{\mathrm{C}}$, can also switch to the high-loss state when a high power signal is injected, because of their nonlinear surface impedance [1]. This property can be used to develop microwave power limiter devices, in which the current flows with high losses when the current density reaches the material-dependent critical current density $J_{c}$ and consequently the signals are attenuated at the output of the limiter.

The advantages of superconducting materials, for the design of microwave power limiters, over other technologies are mainly their short transition period from the superconducting to the normal state, their very low-loss behavior in the

Manuscript received August 03, 2010; accepted November 08, 2010. Date of publication December 23, 2010; date of current version May 27, 2011. This work was supported in part by the Spanish Ministry of Science and Innovation under Grant TEC-2009-13897-C03-01/TCM, by the AGAUR, Generalitat de Catalunya (2008-BE2-00196) and MAT-2008-06761-C03-02; and by Spanish Ministry of Education through PhD fellowships for E. Rocas (BES-2007-16775) and A. Padilla (AP200802235).

E. Rocas, A. Padilla, J. M. O'Callaghan, and C. Collado are with Universitat Politècnica de Catalunya (UPC), Barcelona 08034, Spain.

J. Mateu is with Universitat Politècnica de Catalunya (UPC), Barcelona 08034, Spain. He is also with the Centre Tecnològic de Telecomunicacions de Catalunya (CTTC).

N. Orloff and J. C. Booth are with the National Institute of Standards and Technology, Boulder, CO 80305 USA (e-mail: booth@boulder.nist.gov).

Color versions of one or more of the figures in this paper are available online at http://ieeexplore.ieee.org.

Digital Object Identifier 10.1109/TASC.2010.2093554 superconducting state, and their ability to reversibly switch between low-loss and high-loss states. Previous work has shown that superconducting transmission line limiters can be used to protect downstream electronics from high-power transients, with nanosecond or less turn-on periods [2], [3]. These result in broadband limiters that provide attenuation over the entire frequency band of interest whenever a high-power signal is present. A more desirable approach is to restrict the limiter response to a narrow frequency band around the high-power signal, without degrading the device performance in the remainder of the frequency band. To accomplish this, we implemented a multiplexed superconducting limiter filter bank.

The limiting power in superconducting transmission line limiters was set by the operating temperature and by the cross-section of the implemented transmission line [2], [3], usually resulting in very narrow line-widths (10 $\mu \mathrm{m}$ to $20 \mu \mathrm{m})$. It is expected the use of resonant structures as limiting devices could provide a better control of the limiting power, because additional factors may be used for control, such as quality factor (Q) and coupling between resonators and input and output ports [4]. This approach could also lower the threshold powers significantly compared to those of transmission line limiters.

In this work, we use the measured response of transmission line limiters as the basis for the design of narrowband signallimiting filters at microwave frequencies. We calculate the current density distribution of the individual resonators that constitute the filter from the incident power, as a function of the filter topology, bandwidth, quality factor, and dimensions, and subsequently determine the filter response as a function of incident power. We also studied the detuning of the filter produced by a high power signal and its effect on the overall multiplexer response.

\section{MultiPleXING ARChiteCture}

Fig. 1 outlines a multiplexing architecture in which the incident signal is split into several frequency bands, and subsequently re-combined. Each frequency band, set by a microwave filter, takes only a portion of the incident signal. When the signal power at one or several frequency bands exceeds the limiting power value, set by the critical current density $J_{c}$, the superconducting transmission line resonators switch from the low-loss superconducting state to the high-loss normal state. Therefore, the signal in the high power channel is attenuated and thus the downstream circuitry is protected from the effects of large transient input signals. Because the transition from the superconducting state to the normal state is reversible and occurs on nanosecond timescales, the architecture of Fig. 1 can also be 


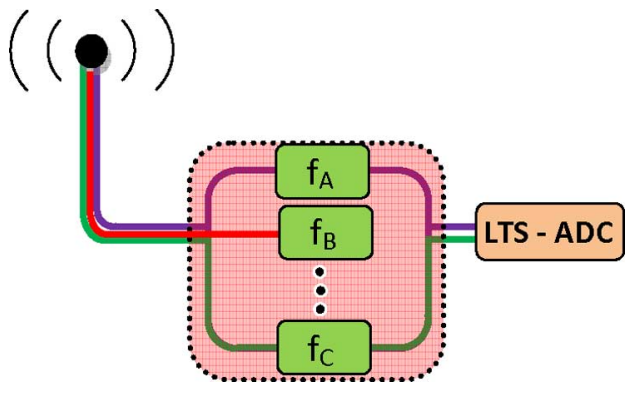

Fig. 1. Outline of the multiplexing architecture, used to protect an analog to digital converter (ADC) from high-power transients.

understood as a high-performance, nanoseconds tunable notch filter.

\section{Limiting POWER AND GEOMETRY IN SUPERCONDUCTOR TRANSMISSION LINES}

The superconducting transmission line limiters reported in [2], [3] essentially consist of typical coplanar waveguides (CPW) transmission line, structured to exceed the critical current density for a given input power level. Such devices were fabricated from a $180 \mathrm{~nm}$ YBCO thin film deposited on an $r$-plane sapphire substrate by pulsed-laser deposition, with a thin $(20 \mathrm{~nm}$ to $50 \mathrm{~nm}) \mathrm{CeO}$ buffer layer. Sapphire substrates were used for their high thermal conductivity and low microwave loss. The measured surface resistance at $3 \mathrm{GHz}$ and $70 \mathrm{~K}$ was approximately in $\mathrm{R}_{\mathrm{S}} \sim 50 \mu \Omega$. The CPW transmission lines had three different center-conductor line widths (55 $\mu \mathrm{m}, 22 \mu \mathrm{m}$, and $11 \mu \mathrm{m})$ and gap spacings (105 $\mu \mathrm{m}, 40 \mu \mathrm{m}$, and $20 \mu \mathrm{m}$, respectively) between the center conductor and ground planes on either side, to achieve a $50 \Omega$ characteristic impedance. Measurements of the limiting behavior revealed an average critical current density at $70 \mathrm{~K}$ of $J_{c}=4.4 \mathrm{MA} / \mathrm{cm}^{2}$.

In this section the material parameters extracted from previous work [2], [3] are used to obtain the saturation power in a different set of CPW transmission lines. Each CPW transmission line has a center conductor that ranges from $10 \mu \mathrm{m}$ to 450 $\mu \mathrm{m}$ wide, and the gap is set to obtain $50 \Omega$ transmission lines. In a $50 \Omega$ superconducting transmission line, matched both with the source and the load, the total peak current $I_{0}$ flowing through a cross-section of the transmission line can be related to the input available power $P_{0}$ by

$$
P_{0}=\frac{I_{0}^{2} Z_{0}}{2}
$$

Note, therefore, that for a given input available power, a whole range of transmission lines of different dimensions would yield the same total currents, as long as the same characteristic impedance is preserved.

The total current is distributed along the cross-section of the transmission line, note that the current density peaks are at the edges of the center conductor and at the inner edges of the ground planes. The current density profile can be calculated by means of numerical techniques such as the Weeks-Sheen method [5], [6]. Fig. 2 displays the maximum current density obtained in the center conductor (in solid line) for an input power

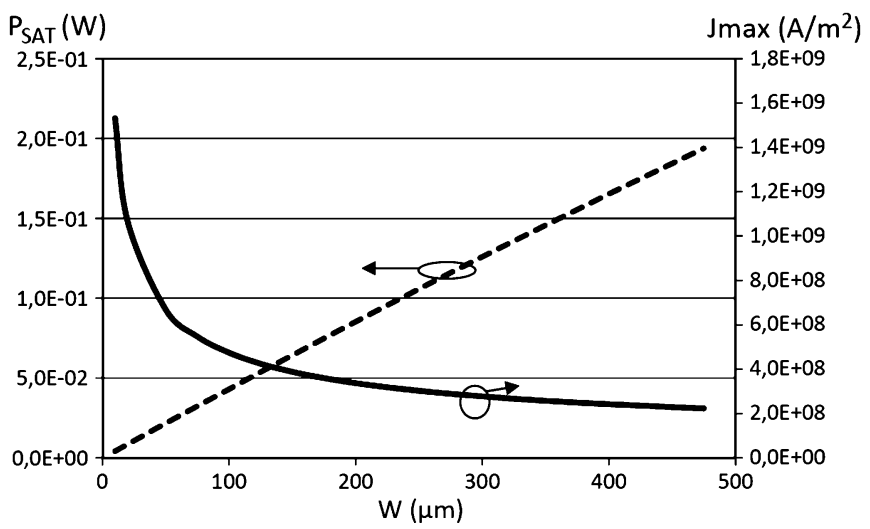

Fig. 2. Dashed line: saturation power as a function of the line width. Solid line: maximum current density as a function of the center conductor width (W), for an input power $\mathrm{P}_{0}=5 \mu \mathrm{W}$.

of $5 \mu \mathrm{W}$, as a function of the center conductor width. As expected, the current density increases for narrower transmission lines. These values are then used, along with (1), to obtain, as a function of center conductor width, the incident power $P_{0}$ that gives a current density that exceeds the critical current density of $J_{c}=4.4 \mathrm{MA} / \mathrm{cm}^{2}$. The results in Fig. 2 (dashed line) show an almost linear dependence of saturation power on the conductor width.

This result shows explicitly that the limiting power in a superconducting transmission line can be set by use of the transmission line width. In the following section, we make use of these material properties to obtain the limiting power for frequency-selective resonators.

\section{SUPERCONDUCTING RESONATOR LIMITERS}

Here we evaluate the saturation power in CPW and microstrip superconducting half-wavelength resonators. Note that, in contrast to the traveling wave transmission line, a resonator need not have a characteristic impedance of $50 \Omega$. This allows us to use microstrip configurations of several center conductor widths, in addition to CPW configurations. In a half-wavelength transmission line resonator the current distribution along the line follows approximately a sinusoidal profile $I(z)=I_{0} \cdot \sin (\pi z / l)$, where $l=\lambda / 2$ and $I_{0}$ is the maximum peak current achieved in the middle of the line. We also know that the $I_{0}$ value depends on the $Q$ factor of the resonator. Hence, high- $Q$ resonators reach high-current values, which in turn results in lower saturation powers.

To achieve high $Q \mathrm{~s}$ in planar technology, designs typically employ relatively wide transmission lines, and concurrently reach high current peaks $\left(I_{0}\right)$. We propose the CPW and microstrip resonator configurations outlined in Figs. 3(a) and 3(b), respectively, which consist of non-uniform transmission lines, where the width of the central region (in grey) is made narrower than the width of the rest of the resonator. Additionally, since the nonlinear behavior in superconductors is a distributed effect [12], this configuration would reduce the nonlinear effects produced in the resonant transmission line.

In non-uniform transmission line resonators the $Q$ factor is

$$
Q_{0}=\omega_{0} \frac{2 L_{d 1} a_{1}+L_{d 2} a_{2}}{2 R_{d 1} a_{1}+R_{d 2} a_{2}},
$$




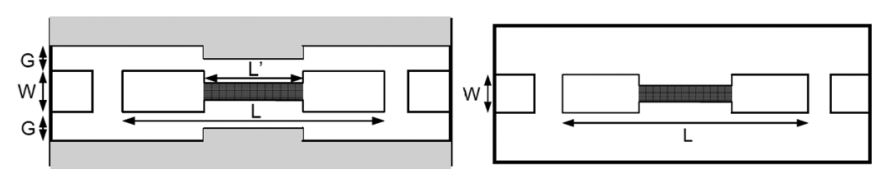

Fig. 3. Outline of the resonators: a) CPW, b) microstrip.

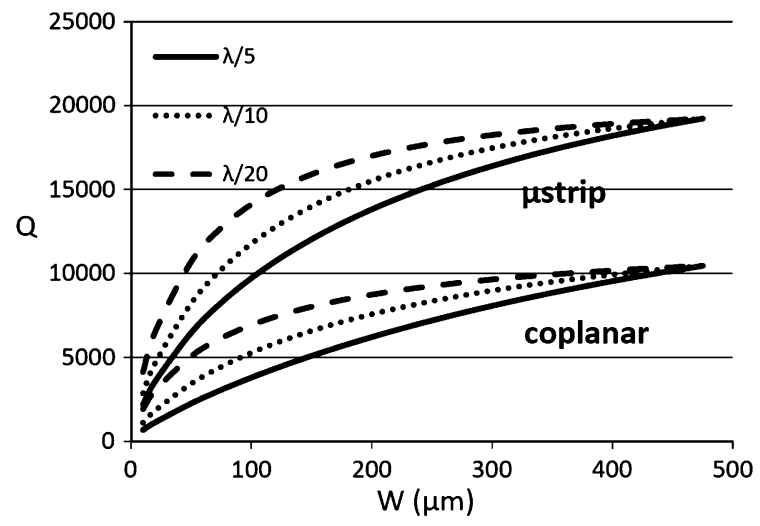

Fig. 4. $Q$ factors as a function of the transmission line width, for microstrip and coplanar resonator configurations and for values of $l_{2}-l_{1}=\lambda / 5, \lambda / 10$ and $\lambda / 20$.

where $L_{d 1}$ and $R_{d 1}$ are the inductance and resistance per unit length, corresponding to the wider region, and $L_{d 2}$ and $R_{d 2}$ are the inductance and resistance per unit length corresponding to the narrower region. The distributed inductance and resistance values can be obtained by means of the Weeks-Sheen method [5], [6], for the widths and thicknesses of each region. The $a_{1}$ and $a_{2}$ terms are geometrical factors, given as $a_{1}=\int_{0}^{l_{1}} \sin ^{2}(\pi z / l) d z$ and $a_{2}=\int_{l_{1}}^{l_{2}} \sin ^{2}(\pi z / l) d z$, respectively, where $2 l_{1}$ is the total length of the wider region of the transmission line resonator of Fig. 3, and $l_{2}-l_{1}$ is the length of the narrower region. Note that when the current distribution differs from a sinusoidal pattern the values of $a_{1}$ and $a_{2}$ have to be recalculated accordingly. The relation between the driven available power $P_{0}$ and the maximum current in the resonator is

$$
P_{0}=\frac{1}{4} I_{0}^{2} \frac{\omega_{0}}{Q_{L}} \frac{(1+2 K)}{2 K}\left(2 L_{d 1} a_{1}+L_{d 2} a_{2}\right),
$$

where $Q_{L}$ is the loaded $Q$ and $K$ the coupling coefficient between the input (output) port and the resonator.

By use of (2), we extract the $Q$ factor for a set of CPW and microstrip resonators as a function of the line width on the center region of the conductor and for several values of $l_{2}-l_{1}(\lambda / 5$, $\lambda / 10$ and $\lambda / 20)$. Note that the wider parts of the transmission lines are set to a fixed value of $450 \mu \mathrm{m}$, which results in $50 \Omega$ regions.

The data of Fig. 4 are used, along with (3), to obtain the maximum current $I_{0}$ and maximum current density $J_{\max }$ for the set of CPW and microstrip resonators. For a critical coupling value $K=0.5$ and for a fixed drive power $P_{0}=5 \mu \mathrm{W}$, the results for CPW and microstrip resonators are summarized in Figs. 5(a) and 5(b), respectively. The maximum current $I_{0}$ increases with the width of the conductor due to the $Q$ dependence, whereas the maximum current density decreases when the center conductor width increases. Additionally, by use of
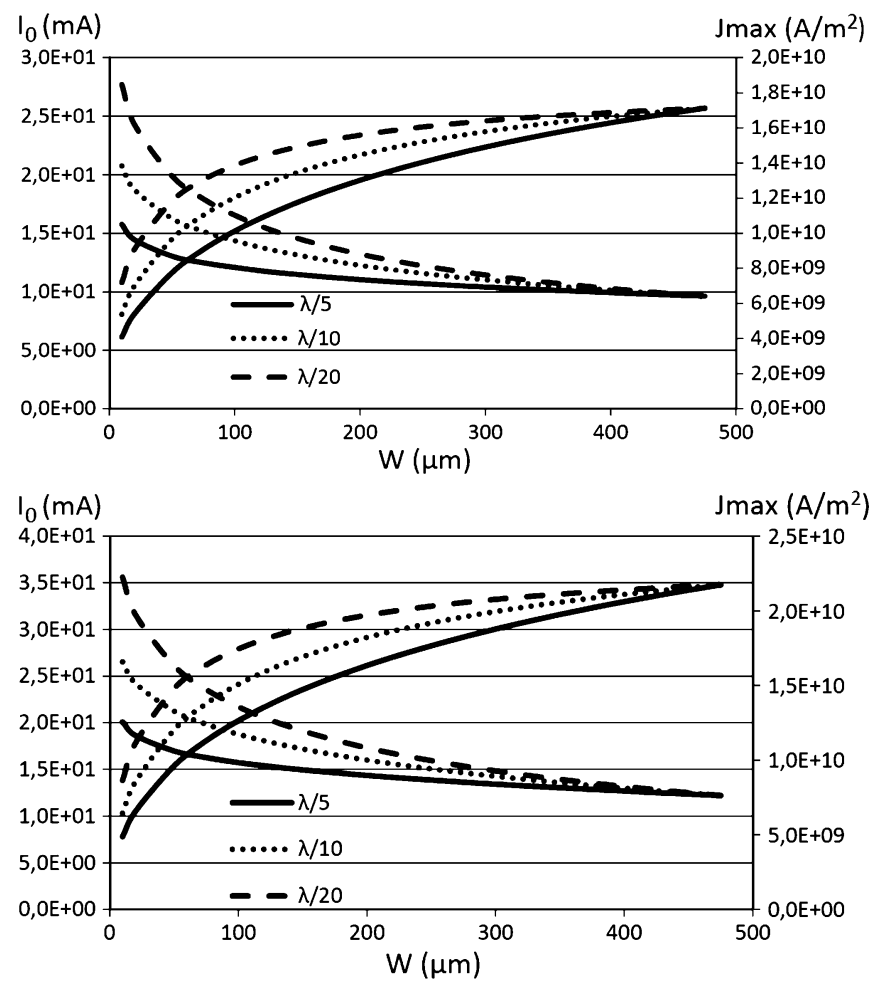

Fig. 5. Maximum current $I_{0}$ (left ordinate) and maximum current density $J_{\max }$ (right ordinate) as a function of the conductor width and for several values of $l_{2}-l_{1}=\lambda / 5, \lambda / 10$ and $\lambda / 20$. (a) CPW; (b) microstrip.

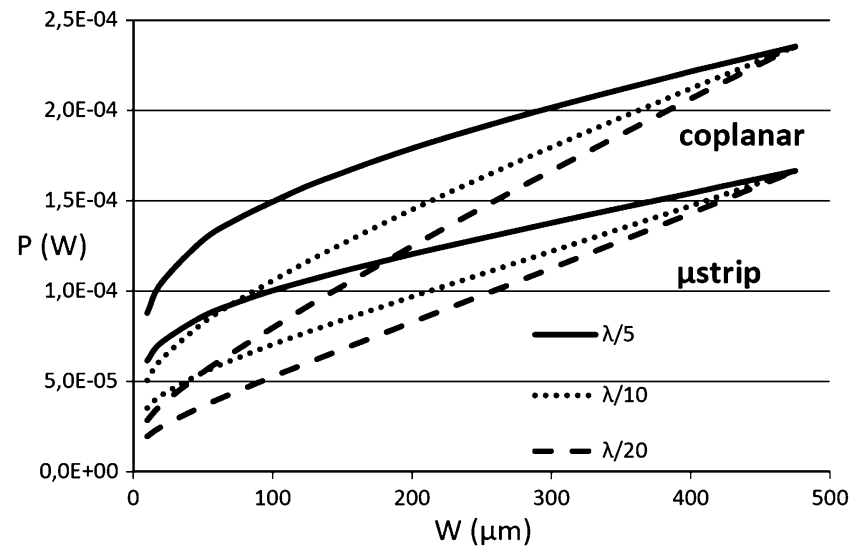

Fig. 6. Saturation power as a function of the conductor width and for $l_{2}-l_{1}=$ $\lambda / 5, \lambda / 10$ and $\lambda / 20$, for CPW and microstrip.

(3), we also obtain the saturation power for the resonant structures presented above. For the CPW and microstrip resonators, the results are shown in Fig. 6. Note that the saturation power is reduced considerably compared to the case for the transmission line limiters.

\section{POWER Limiting SuPERCONDUCTOR FILTER BANK}

Next, we need to find the total current flowing through the filter resonators, as was previously done to obtain the saturation power, so that the total current can then be linked to the current density distribution. The resonant structures assumed for this investigation are those presented in the previous section, and their $Q$ factors are summarized in Fig. 4. 


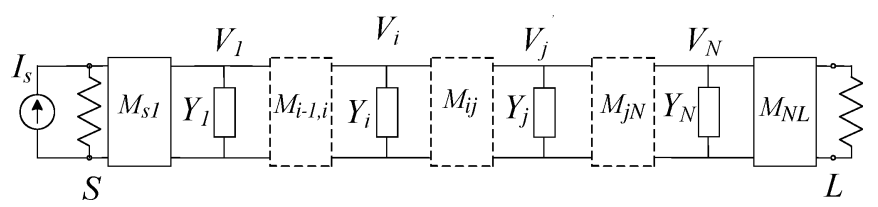

Fig. 7. Outline of the filter circuit model made of in-line coupled resonators.

We start by considering a circuit model of a filter made of $N$ coupled resonators (see Fig. 7), which can be formulated with its general $N \times N$ admittance matrix $Y$ [7]. Note that for clarity Fig. 7 corresponds to an in-line filter configuration. Nevertheless, the formulation below is general for any filter topology. The admittance lumped elements $Y_{i}$ correspond to the RLC parallel resonator model [8]. The admittance matrix is then used to obtain the voltage $V_{i}$ at each node of the equivalent-circuit model as a function of the current source $I_{s}$, which is related to the driven power $P_{0}=I_{s}^{2} Z_{0} / 8$. The resulting dissipated power is

$$
P_{d}=\frac{V_{i}^{2}}{2 R_{i}}
$$

where $R_{i}$ is the lumped resistance that accounts for the resonator losses and can be related to the quality factor as $Q_{0}=R_{i} / \omega L_{i}$, where $L_{i}$ is the inductance of the RCL resonator model [8]. The filter configurations assumed in this section use the last resonator in the filter network as the limiting resonator. Note however that the formulation and results could be extended for any other location of the limiting resonator or even when more than a limiting resonator is used. The final position of the limiting resonator would depend on the multiplexing architecture used in the prototype, not yet properly addressed in this work.

The dissipated power at each resonator can also be obtained from the current distribution at each resonator, for half-wavelength resonators, the current distribution at the ith resonator is $I(z)=I_{0 \_i} \sin (\pi z / l)$. Then, the resulting dissipated power can be obtained as:

$$
P_{d}=I_{0}^{2}\left[2 R_{d 1} a_{1}+R_{d 2} a_{2}\right]
$$

By equating (4) and (5), we can extract the maximum current at each resonator, which is used to obtain the maximum current density and therefore the saturation power. By doing that, we can find the saturation power for several filters, of different order $(N)$ and bandwidth (BW). Fig. 8 shows the saturation power in a fourth order Chebyshev filter with return losses of $20 \mathrm{~dB}$, centered at $3 \mathrm{GHz}$ and for $20 \mathrm{MHz}$ and $200 \mathrm{MHz}$ bandwidths, as a function of the center conductor width.

The results reveal that the required power to saturate the filter decreases when the filter bandwidth decreases and approaches that required in a single resonator for very narrow band filters. This conclusion might be very significant from a practical point of view, since we can set the limiting power by designing filters of different bandwidths. In addition, Fig. 9 shows the saturation power for a Chebyshev filter (with $20 \mathrm{~dB}$ return losses) for several orders, $\mathrm{N}=3,4,6$ and 8 . In this case, we can be observed that the saturation power is largely independent of the order of the filter.

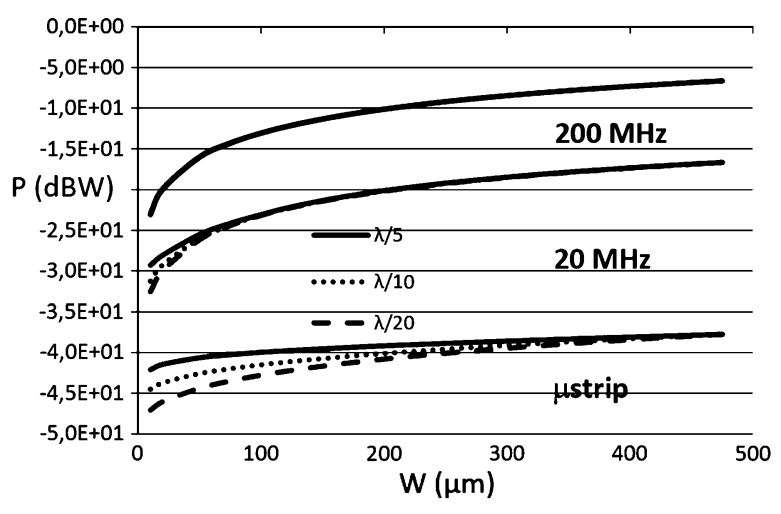

Fig. 8. Saturation power for 4th order Chebyshev filters of $20 \mathrm{MHz}$ and 200 $\mathrm{MHz}$ bandwidth. The saturation power for a single microstrip resonator is also depicted for comparison.

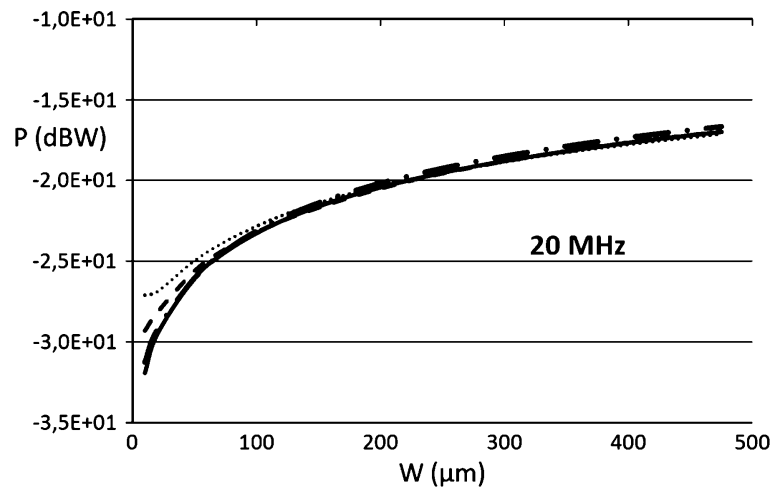

Fig. 9. Saturation power for third-, fourth-, sixth- and eighth-order Chebyshev filters with $20 \mathrm{MHz}$ bandwith. Dotted: eighth, dashed: sixth, dash-dotted: fourth, and solid: third.

\section{PRACTICAL CONSIDERATIONS ON THE IMPLEMENTATION OF A SUPERCONDUCTING POWER LIMITER MULTIPLEXER}

The formulation and procedure presented here allow us to extract the limiting power of a superconducting filter by obtaining the total current that flows through the resonators when the maximum current density exceeds the critical current density. However, the inherent nonlinear nature of superconductors [9], which gives rise to the limiting behavior, can also produce effects such as detuning and saturation at high current densities. Therefore, we may expect a variation of the resonant frequency and reduction in $Q$ for the resonators in the filter as the current density increases. The quantification of the detuning and $Q$ reduction can be obtained by means of modeling the nonlinear distributed effects of the superconducting resonators [9]-[13].

Assessment of the detuning effects and $Q$ reduction in a single filter is necessary when the filter is used in a multiplexing architecture, because an out-of band variation of the input reflective coefficient may adversely affect the entire system performance [7]. Fig. 10 shows the above-mentioned effects in a sixth-order Chebyshev filter centered at $3 \mathrm{GHz}$ with a $200 \mathrm{MHz}$ bandwidth, and whose initial Q values are set to $10^{4}$. Figs. 10(a) and 10(b) show the frequency response of the filter when the $Q$ of the last resonator is reduced from $10^{4}$ to $10^{3}$ and $10^{2}$, respectively. The results show that the input reflection coefficient remains fully reflective when out-of-passband, 

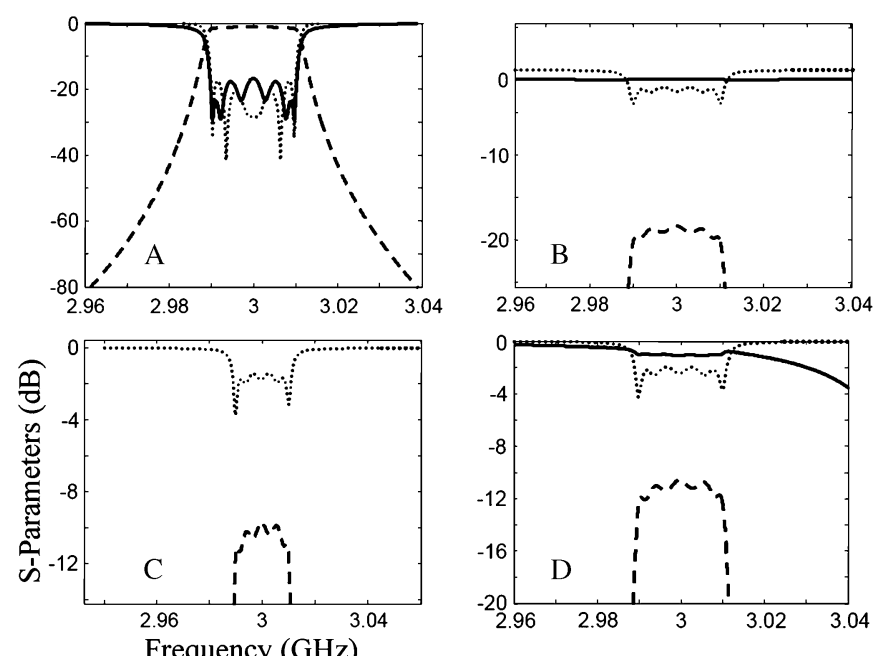

Fig. 10. Filter frequency response. (a) the $\mathrm{Q}$ of the last resonator is reduced from 10,000 to 1,000 ; (b) the $\mathrm{Q}$ of the last resonator is reduced from 10,000 to 100 ; (c) the last resonator is detuned by $2 \%$, maintaining a $\mathrm{Q}$ of 10,000 ; and (d) the last resonator reduces the $\mathrm{Q}$ from 10,000 to 100 and detuned $2 \%$. The pink line corresponds to $S_{21}$, the blue line to $S_{11}$ and the black line to $S_{22}$.

and the transmission coefficient suffers additional insertion loss. Fig. 10(c) shows the frequency response when the last resonator is detuned by $2 \%$. When the detuning of the last resonator occurs, there is still a fully reflective input coefficient. Finally, in Fig. 10(d) we show the effects of a reduction of $Q$ (from $10^{4}$ to $10^{2}$ ) and detuning of the last resonator by $2 \%$. From the results of Fig. 10 we may therefore conclude that the nonlinear effects occurring in the superconducting filters do not degrade the overall performance of the multiplexing scheme, and will only produce attenuation of the signal passing through the corresponding filter.

The nonlinear effects also produce spurious signals which may reduce the ADCs dynamic range. Although this is not explicitly addressed in this work, the short narrow limiting section in the middle of the transmission line forecast a low value of intermodulation signals [12].

\section{CONCLUSION}

This work evaluated the use of a superconducting multiplexer filter bank to operate as a frequency-selective power limiter. In order to achieve this functionality, we have formulated the saturation power of the resonators and filters as a function of the superconducting material properties, geometry of the resonators, and the parameters defining the filters, such as the filter bandwidth and filter order. We then used this formulation to calculate the limiting power in several filter configurations. We have used the material parameters extracted from measurements on transmission-line limiters [2]. We conclude that the limiting power in a multiplexing architecture would be much lower than that required in a regular transmission line limiter. The limiting power can be controlled by the dimensions of the resonators that constitute the filters, the operating temperature, and the filter bandwidth. Analysis of the nonlinear effects occurring in a superconducting device shows that the overall performance of the multiplexing structure would not be degraded by the nonlinearities.

\section{REFERENCES}

[1] Z. Y. Shen, High-Temperature Superconducting Microwave Circuit. Boston: Artech House, 1994.

[2] J. C. Booth, K. Leong, and S. A. Schima, "A superconducting microwave power limiter for high performance receiver protection," in IEEE Trans. Microw. Theory and Tech.-Symposium Digest, 2004, pp. 139-142.

[3] J. C. Booth, D. A. Rudman, and R. H. Ono, "A self-attenuating superconducting transmission line for use as a microwave power limiter," IEEE Trans. Applied Supercond., vol. 13, no. 2, pp. 305-310, June 2003.

[4] C. Collado, J. Mateu, J. C. Booth, and J. M. O'Callaghan, "Detuning and saturation of superconducting devices: Formulation and measurements," in IEEE Trans. Microw. Theory and Tech.-Symposium Digest, 2007, pp. 2023-2026.

[5] W. T. Weeks, L. L. Wu, M. F. McAllister, and A. Singh, "Resistive and inductive skin effect in rectangular conductors," IBM J. Res. Dev., vol. 23, pp. 652-660, 1979.

[6] D. M. Sheen, S. M. Ali, D. E. Oates, R. S. Whiters, and J. A. Kong, "Current distribution, resistance, and inductance for superconducting strip transmission lines," IEEE Trans. Appl. Supercond., vol. 1, pp. 108-115, June 1991.

[7] R. J. Cameron, C. M. Kudsia, and R. R. Mansour, Microwave Filters for Communication systems. Fundamentals, Design, and Applications. : John Wiley \& Sons, 2007.

[8] D. Pozar, Microwave Engineering. : John Wiley \& Sons, Inc., 1998.

[9] T. Dahm and D. J. Scalapino, "Theory of intermodulation in superconducting microstrip resonator," J. Appl. Phys., vol. 81, no. 4, pp. 2002-2012, 1997.

[10] J. C. Booth, J. Bell, D. Rudman, L. Valle, and R. Ono, "Geometry dependence of nonlinear at microwave frequencies," J. Appl. Phys., vol. 86, no. 2, pp. 1020-1025, 1999.

[11] J. C. Booth, K. Leong, S. A. Schima, C. Collado, J. Mateu, and J. M. O'Callaghan, "Unified description of nonlinear effects in high temperature superconductors," Journal of Supercond., 2006, 10.1007/s10948006-0126-2, published online.

[12] C. Collado, J. Mateu, and J. M. O'Callaghan, "Analysis and simulation of the effects of distributed nonlinearities in microwave superconducting devices," IEEE Trans. Appl. Supeconduc., vol. 15, no. 1, Mar. 2005.

[13] J. Mateu, J. C. Booth, C. Collado, D. Seron, and J. M. O’Callaghan, “Intermodulation distortion in coupled-resonators filters with nonuniform distributed properties: Use in HTS IMD compensation," IEEE Trans. Microw. Theory and Tech., vol. 54, pp. 1154-1160, Mar. 2006. 